\title{
Measles Data Reporting in the District Health Information System: A Case Study of Gombe State
}

\author{
Nnamdi Usifoh ${ }^{1}$, Toby Yak $^{1}$, Ivy Dooga ${ }^{2}$, Raymond Dankoli ${ }^{3}$, Olufemi Ajumobi ${ }^{2}$, \\ Adewole Adefisoiye $^{2} \&$ Oluwasegun Joel Adegoke ${ }^{2}$ \\ ${ }^{1}$ University of Roehampton, London, United Kingdom \\ ${ }^{2}$ African Field Epidemiology Network, Abuja, Nigeria \\ ${ }^{3}$ Gombe State Ministry of Health, Gombe, Nigeria \\ Correspondence: Nnamdi Usifoh, AFENET Nigeria, 50 Haile Selassie Street, Asokoro, Abuja, Nigeria. Tel: \\ 234-805-431-6199. E-mail: maxiusifoh@gmail.com
}

Received: July 30, 2019 Accepted: September 9, 2019 Online Published: September 17, 2019

doi:10.5539/gjhs.v11n11p109 URL: https://doi.org/10.5539/gjhs.v11n11p109

\begin{abstract}
Background: The District Health Information System (DHIS2) is a modular, cloud-based data management system designed for use in integrated health information systems. In Nigeria, it serves as the repository for routine health data, including measles. A first dose of measles is given routinely in most countries, however, for a country to include a second dose of measles in the routine immunization schedule, it must meet certain criteria set by the World Health Organization (WHO). Unfortunately, Nigeria falls into the category of countries that haven't met the criteria. Despite this, MCV2 data can be seen on the DHIS2 platform. Data from DHIS2 also shows that Gombe State has the highest number of health facilities that reported MCV2 data at least once from 2015 to 2017.

The aim of the study was to determine the reasons for the MCV2 reporting on DHIS2 platform for Gombe State.

Method: We conducted a cross-sectional study among health workers in selected health facilities and LGA RI Officers at the LGA level in Gombe State. Health facility registers were reviewed, and data consistency was ascertained. We reviewed and conducted secondary data analysis of MCV2 data for Gombe State from January 2015 to December 2017.

Results: Of the 22 health facilities assessed, 14 health facilities (12 public and 2 private) reported offering MCV2 during the health facility-level interviews. At the LGA level, 5 LGAs out of the 11 LGAs reported during the LGA-level interviews that a second dose of measles is part of the RI schedule in their respective LGAs. For the 6 LGAs that reported not offering a second dose of measles as part of the RI schedule, 3 LGAs identified data entry error as the possible reason for having MCV2 data in the DHSI2 platform while the remaining 3 LGAs reported that the MCV2 data in the DHIS2 platform can be attributed to recording children who didn't receive a first dose of measles at 9 months but received at 18-23 months as second dose of measles.
\end{abstract}

Conclusion: Data entry error and knowledge gap on how to record measles data were identified factors responsible for MCV2 data on the DHIS2 platform. There is a need for targeted interventions towards improving the quality of RI data in Nigeria.

Keywords: measles, DHIS2, routine immunization 


\section{List of Abbreviations}

$\begin{array}{ll}\text { BCG } & \text { Bacillus Calmette Guerin } \\ \text { CCO } & \text { Cold Chain Officer } \\ \text { DHIS2 } & \text { District Health Information System version 2 } \\ \text { DPT } & \text { Diphtheria Pertussis and Tetanus } \\ \text { DQA } & \text { Data Quality Assessment } \\ \text { DVD MT } & \text { District Vaccination Data Management Tool } \\ \text { FMoH } & \text { Federal Ministry of Health } \\ \text { HF } & \text { Health Facilities } \\ \text { HMIS } & \text { Health Management Information System } \\ \text { IBM SPSS } & \text { International Business Machines Statistical Package for the Social Sciences } \\ \text { LGA } & \text { Local Government Area } \\ \text { LIO } & \text { Local Government Immunization Officer } \\ \text { LGA } & \text { Local Government Area } \\ \text { M\&E } & \text { Monitoring \& Evaluation } \\ \text { MCV } & \text { Measles Containing Vaccine } \\ \text { MICS } & \text { Multiple Indicator Cluster Survey } \\ \text { NCH } & \text { National Council on Health } \\ \text { NICS } & \text { National Immunization Coverage Survey } \\ \text { ODK } & \text { Open Data Kit } \\ \text { PHC } & \text { Primary Healthcare Centres } \\ \text { QI } & \text { Quality Improvement } \\ \text { RI } & \text { Routine Immunization } \\ \text { SIA } & \text { Supplemental Immunization Activities } \\ \text { SIO } & \text { State Immunization Officer } \\ \text { UNICEF } & \text { United Nations Children's Fund } \\ \text { VPD } & \text { Vaccine Preventable Disease } \\ \text { WHO } & \text { World Health Organization } \\ & \end{array}$




\section{Introduction}

One-third of children in many African countries get infected with measles within the first 2 years of life (Ibrahim, Usman, Muhammed, \& Okunromade, 2017). Globally, measles-related deaths have decreased by $80 \%$ from 853,479 in 2000 to 173,330 in 2017 , with the incidence decreasing $83 \%$, from 145 to 25 cases per million population (Dabbagh et al., 2018). However, in Africa, measles remains one of the leading causes of morbidity and mortality. These high case-fatality rates can be linked to infection at a young age, poor shelter and overcrowding, underlying immune deficiency disorders due to malnutrition, vitamin A deficiency, and lack of access to medical care (Ibrahim et al., 2017). An estimated 20.8 million children are still missing their first dose of measles vaccines and half of these children live in Nigeria, Pakistan, India, Indonesia, Ethiopia and the Democratic Republic of Congo (DRC), with Nigeria having the highest number of unvaccinated children among the six countries (3.3 million) (Measles \& Rubella Initiative, 2017).

While Measles Containing Vaccine first dose (MCV1) has been given routinely in most countries at 9-12 months of age, WHO recommends that Measles Containing Vaccine second dose (MCV2) be included in the routine immunization schedule of countries that have attained $\geq 80 \%$ coverage of MCV1 nationally for at least 3 years (WHO, 2009). Globally, 194 countries have introduced MCV2 into the national schedule (Biellik \& Davis, 2017). In Nigeria, MCV2 is yet to be included in the national Routine Immunization (RI) schedule, having not attained the required national coverage of $\geq 80 \%$ for MCV1. An additional dose is usually administered as a booster dose during measles Supplemental Immunization Activities (SIAs)/campaigns. The data generated from campaigns is captured separately from routine data generated from regular immunization sessions in the health facilities, using data tools customized specifically for the campaign.

In Nigeria, routine data for measles is entered into the District Health Information System (DHIS2). Health data is aggregated at the Local Government Area (LGA) level. Health facilities submit hard copies of summary forms for the month to the LGA team for entry into the DHIS2 platform. Since MCV2 hasn't been officially introduced into the routine immunization module, it is expected that data collected for children immunized with measles vaccines will be recorded as MCV1, however, some health facilities have captured some of the data as MCV2 on the DHIS2 platform. Recent scrutiny of the measles data on the platform by the study team suggests that data is being captured for MCV2 in $667(86 \%)$ out of 774 LGAs in the country, thus raising concerns about the quality and validity of measles data generated from the service delivery points.

Between 2015 and 2017, Gombe State has recorded high administrative coverage for measles, however, the results of the recently conducted 2016/2017 Multiple Indicator Cluster Survey (MICS)-National Immunization Coverage Survey (NICS) showed very low coverages for measles and other key antigens, thus raising questions about the quality of routine immunization administrative data captured on the DHIS2 platform. In 2017, the national administrative coverage of MCV1 was pegged at $104 \%$ while the survey data showed a coverage of $42 \%$, with Gombe posting a survey coverage of $32.4 \%$ (UNICEF, 2018). Gombe State was selected for this study because from 2015-2017, the State recorded the highest number of health facilities that reported MCV2 data at least once on the DHIS2 platform. The study aimed at determining reasons for MCV2 reporting on the DHIS2 platform among healthcare workers in Nigeria.

There is little evidence of studies conducted to evaluate the quality of measles data inputted into the DHIS2 platform. Most studies on routine immunization data quality are centered around the level of routine immunization data consistency between reporting forms at the lower levels of service delivery (health facility, ward, LGA) however, several studies on routine immunization data quality and reporting from health facilities offering routine immunization services. Some of these studies have been able to link the poor quality of RI data to the mechanisms involved in data collection, analysis and use at the lower levels of service delivery.ie. health facility level. One of such studies was the assessment of routine immunization data reporting and quality in Bunza LGA, Kebbi State (Omoleke \& Tadesse, 2017). This study focused on the poor quality of routine immunization administrative data which has affected the responses to Vaccine Preventable Diseases (VPDs). The researchers conducted a cross-sectional study in selected health facilities in Bunza LGA, using semi-structured questionnaires and survey tools for primary data collection. Data consistency checks for Penta 3 doses administered in September 2016 were conducted between tally sheets, RI registers, health facility summary forms and LGA summary forms. RI providers and programme managers were also interviewed to ascertain the possible reasons for the discrepancies in data among the reporting forms. Results from the study also showed huge discrepancies between the data for selected antigens across the reporting forms that were scrutinized. Suggested methods for improving data quality were improved supportive supervision to health facilities, improved community linkages, among others (Omoleke \& Tadesse, 2017). 
Kiberu et al. (2014) evaluated the experience of Uganda in strengthening routine health data reporting through the roll-out of the District Health Management Information System version 2 (DHIS2). The platform was launched in Uganda in 2011 to replace the paper-based Health Management Information System (HMIS) that had been used for years (Kiberu et al., 2014). The roll-out process included 35 training workshops for 972 participants/users across the country. To assess improvements in health reporting in Uganda, the researchers compared data on completeness and timeliness of outpatient and inpatient reporting for the period before and after the introduction of DHIS2. Data on selected health service coverage indicators were collected, while implementation challenges and lessons learned during the DHIS2 roll-out process were also documented. Results of the study showed that the completeness of outpatient reporting increased from $36.3 \%$ in $2011 / 12$ to $85.3 \%$ in 2012/13 while timeliness of outpatient reporting increased from $22.4 \%$ to $77.6 \%$; completeness of inpatient reporting increased from $20.6 \%$ to $57.9 \%$ while timeliness of inpatient reporting increased from $22.5 \%$ to 75.6 . Documented Implementation challenges included limited access to computers and internet (34\%), inadequate technical support (23\%) and limited worker force (18\%). By the end of the evaluation, the researchers concluded that Implementation of DHIS2 in Uganda resulted in improved timeliness and completeness in reporting of routine outpatient, inpatient and health service usage data from the district to the national level. They also recommended that continued onsite support supervision, mentorship and additional system/infrastructure enhancements, including internet connectivity, are needed to further enhance the performance of DHIS2, thereby improving the quality and use of health data in the country.

Ohiri et al. (2016) assessed the availability, quality and use of data in the malaria program for decision making in Nigeria, focusing on Akwa Ibom, Cross River and Niger States. The study identified DHSI2 as the reporting platform for health program data, including malaria but also identified gaps in terms of the number of malaria indicators being tracked on the platform. The researchers evaluated data availability by accessing malaria data on the internet and DHIS2 for completeness and timeliness; data quality was assessed by conducting Data Quality Assessment (DQA) in selected health facilities in the identified states and comparing malaria data collected using the HMIS tools and the data entered into DHIS2; data use was evaluated by conducting semi structured interviews with participants selected from different levels of the malaria program in the study States The quality of data assessed was found to be sub-optimal compared to the national benchmark. The study is relevant because it highlights the importance of quality data for decision-making, towards achieving the elimination of malaria in Nigeria, as demonstrated in countries such as Malaysia, Cape Verde, Philippines and others.

A study was conducted by Nsubuga et al. (2018) to assess the quality of immunization data in Karabole District, Uganda. The researchers conducted data quality audits for Diphtheria, Pertussis and Tetanus (DPT) data reported at the district level against the data recorded in the health facilities between July and August 2016. Consistency was estimated using a Verification Factor (VF) that was calculated based on DPT data recounted at the health facilities and the data reported at the district level. Results of the study showed average data consistencies at the health facility level, with varying levels of reporting. The researchers recommended that the Ministry of Health in Uganda focus on improving the quality of immunization data at the lowest levels of health service delivery in the country.

An assessment of the quality of immunization data quality in Tshishimbi Health Zone, Democratic Republic of Congo (Nzaji et al., 2019). The study also focused on the use of DQA tools to assess data accuracy and consistency in four selected health facilities. Results showed over-reporting for measles antigen into two of the selected health facilities, with under-reporting of the same antigen identified in the other facilities. The Quality Index (QI) for the district and health facilities was discovered to be lower than the set threshold of $80 \%$. The researchers highlighted the importance of having quality immunization data to improve the interventions targeted at combating Vaccine Preventable Diseases (VPDs).

A similar study was conducted to assess the quality of routine immunization data monitoring systems in two South-Eastern districts of Anambra State, Nigeria (Fatiregun, 2013). The researchers based their research on the fact that there have been huge discrepancies between data recorded from community surveys and coverage levels from administrative data. The study was conducted in health facilities offering RI in Ogbaru and Onitsha North LGAs (districts) which are rural and urban respectively. These LGAs were randomly selected from a pool of LGAs that hadn't participated in any previous audit. The researchers assessed the consistency of data recorded for DPT3 doses administered and measles in the tally sheets and the data reported on the LGA immunization summary. The study also evaluated data archiving and analysis. Data accuracy was correlated with the age of the health facility routine immunization focal person(s), their knowledge of the RI data tools, attitude of health workers, among others. The study highlighted the challenges with immunization data quality in terms of data inconsistencies, knowledge gaps and staff attitude. 
Githinji et al. (2017) assessed the completeness key malaria indicators reported by health facilities in Kenya in the DHIS2 platform within 5 years of its implementation in the country. The researchers extracted data for key malaria indicators for 6235 public health facilities and 3143 private health facilities from January 2011 to December 2015. Results of the study showed that the completeness of reporting of new antenatal care clients increased from 53.7 to $70.5 \%$ ( $p$ value of $<0.0001$ ) while the completeness of reporting of Intermittent Preventive Treatment in pregnancy (IPTp) decreased from 64.8 to $53.7 \%$ (p value of $<0.0001$ ) for dose 1 and from 64.6 to $53.4 \%$ (p value of $<0.0001$ ) (Githinji et al., 2017) for dose 2. For the private health facilities, completeness of reporting increased significantly for confirmed malaria cases across all age categories i.e. 16.7$23.1 \%$ ( $\mathrm{p}$ value of $<0.0001$ ) in children aged $<5$ years and $19.4-28.6 \%$ ( $\mathrm{p}$ value of $<0.0001$ ) in persons aged $\geq 5$ years (Githinji et al., 2017). In terms of reporting for new ANC clients, completeness improved from 16.2$23.6 \%$ ( $\mathrm{p}$ value $<0.0001$. In 2015 , less than $3 \%$ of data values for malaria tests performed were reported in DHIS2 from the private sector. The implementation of DHIS2 in Kenya resulted in a remarkable increase in the completeness of reporting foe key malaria indicators (completeness of reporting is one of the key dimensions of data quality).

Even though these studies were able to identify gaps in RI data consistency at the lower levels of service delivery and evaluate health data reporting mechanisms, little emphasis was placed on measles data reporting, with most studies on immunization data reporting focusing more on DPT as a proxy for immunization data quality.

\section{Methods}

\subsection{Study Area}

Gombe State is in the North-East region of Nigeria. The State has a total population of 3,328,595, with 615 health facilities spread across 11 Local Government Areas, of which 504 offer RI services (DHIS2, 2018). Between 2015 and 2017, Gombe State recorded high administrative coverage for measles, however, results from recently conducted 2016/2017 Multiple Indicator Cluster Survey (MICS)-National Immunization Coverage Survey (NICS) showed very low coverages for key antigens in the State.

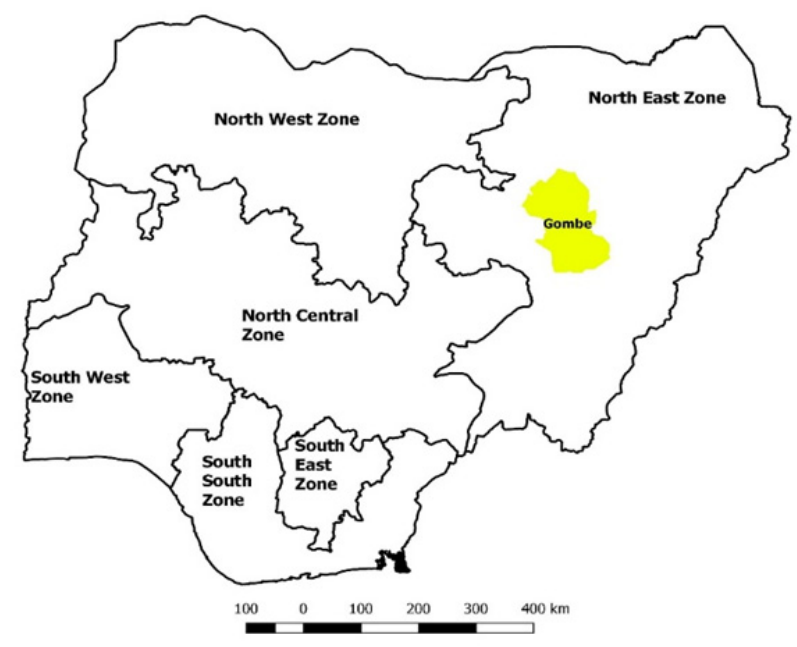

Figure 1. Map of Nigeria depicting the 6 geopolitical zones and the location of Gombe State in the North East Zone

\subsection{Study Design}

We conducted a cross-sectional study among health workers in selected health facilities and LGA RI Officers at the LGA level in Gombe State. Checklists were administered to health workers at the health facility and LGA level to determine the reasons for MCV2 reporting on DHIS2.

\subsection{Study Population Enrolment}

RI officers at the health facility and LGA levels that consented to be interviewed were selected. The number of respondents at the LGA level was 1 per LGA and at the health facility level was 1 per health facility. The selection of respondents was based on knowledge of the subject matter and availability. The designations are as follows: 
Health facility level: Health facility RI focal person

LGA level: Local Government Immunization Officer (LIO), Monitoring \& Evaluation Officer or the Cold Chain Officer in each of the 11 LGAs.

A secondary data analysis of MCV2 data in the DHIS2 platform was conducted to identify health facilities within the 11 Local Government Areas in Gombe State that have consistently reported MCV2 from 2015 to 2017. Then, the health facilities were ranked according to frequency of MCV2 reporting and the quantity of MCV2 data per month from 2015 to 2017 . Within the identified LGAs, all health facilities that reported MCV2 data over the past one year were contacted, from which two health facilities per LGA were selected based on availability of the health facility staff and the willingness to participate in the study. Overall, a total of 22 health facilities were assessed across the 11 LGAs in the State ( 2 health facilities per LGA), of which 19 health facilities were public health facilities while 3 health facilities were private health facilities. Each health facility RI officer in the selected health facilities $(\mathrm{n}=22)$ was interviewed.

\subsection{Data Collection}

Semi-structured questionnaires created on Open Data Kit platform and configured on android-based phones were interviewer-administered to the respondents at the health facility and LGA levels. The questionnaire comprised questions that cut across the following thematic areas: Identification, Measles data quality \& use, Capacity development. At the health facility level, data validation checks were conducted across the RI tally sheets, health facility immunization summary forms and MCV2 data on the DHIS2 platform to identify the areas where the MCV2 reporting discrepancies originated from.

\subsection{Data Processing \& Analysis}

We developed a codebook that contained codes for demographics, including highest education level, and designation. Data was cleaned and recoded using the codebook, for data analysis. Data analysis was conducted using the Statistical Package for Social Sciences (SPSS) software version 21 and Microsoft Excel. Frequencies and proportions of respondents' socio-demographic characteristics and health facility ownership (public and private) were presented using tables and charts. Association was established between independent (health facility ownership) and dependent (availability of data tools, source of measles 2 data, reasons for reporting measles 2 data, levels of trainings attended on RI data quality and use) variables. Level of significance was set at $95 \%$ confidence interval.

\section{Results}

\subsection{Measles Data Quality \& Use}

Table 1 shows the response of the health facilities to whether they offer a second dose of measles:

Table 1. Health facilities that offer a second dose of measles in Gombe State (January 2015 - December 2017)

\begin{tabular}{lccc}
\hline & & \multicolumn{2}{c}{ Health Facility Ownership (n=22) } \\
\cline { 3 - 4 } & & Private Health Facility & Public Health Facility \\
\hline \multirow{2}{*}{ Does this Facility offer a second dose of measles } & No & 1 & 7 \\
& Yes & 2 & 12 \\
\hline
\end{tabular}

Table 2 shows the response of the health facilities to how data for a second dose of measles is recorded:

Table 2. Health facilities that reported offering second dose of measles vaccines in Gombe state (January 2015 December 2017) and how the data is recorded in the registers

\begin{tabular}{lcc}
\hline Schedule & $\begin{array}{c}\text { Private health facility } \\
\text { n=2 }\end{array}$ & $\begin{array}{l}\text { Public health facility } \\
\mathbf{n = 1 2}\end{array}$ \\
\hline $\begin{array}{l}\text { 12-23months } \\
\text { 12-23months who have already received a first dose of measles }\end{array}$ & 0 & $1(8.3 \%)$ \\
$\begin{array}{l}\text { Under children aged 18-23 months who didn't receive a first dose at age } \\
9 \text { months }\end{array}$ & 0 & $2(16.7 \%)$ \\
$\begin{array}{l}\text { Under children aged 18-23 months who have already received a first } \\
\text { dose at age 9 months }\end{array}$ & $2(100 \%)$ & $7(58.3 \%)$ \\
\hline
\end{tabular}


In terms of procuring of vaccines for measles administration, all 12 public health facilities and 2 private health facilities that reported offering a second dose of measles reported receiving the vaccines from the LGA immunization programme and reported using the same stock of first dose of measles vaccines to administer a second dose of measles.

All 22 health facilities (19 public and 3 private) reported recording MCV2 data during Measles campaigns/SIAs as part of part of campaign data, using the data tools prepared specifically for use during the campaigns.

At the LGA level, all the LGA respondents said data is reviewed before it is captured in the DHIS2 platform. Table 3 below shows the number of LGAs that reported offering a second dose of measles and the response and the possible reasons for reporting a second dose of measles, as identified by the LGAs that reported not offering it.

Table 3. LGAs that offer a second dose of measles, and the possible reasons for reporting it (as identified by the LGAs that reported not offering a second dose)

\begin{tabular}{lcc}
\hline LGA offers a second dose of measles $(\mathbf{n}=\mathbf{1 1})$ & Frequency & Percent $(\%)$ \\
\hline Responses & 5 & 45 \\
Yes & 6 & 55 \\
No & 3 \\
\hline Possible reasons for reporting a second dose of measles $(\mathbf{n}=\mathbf{6})$ & 3 \\
\hline Children who didn't receive a first dose of measles at 9 months but received at 18-23 months & 50 \\
Data entry error & 50 \\
\hline
\end{tabular}

Figure 2 below shows the response of the LGAs that reported having a second dose of measles in their RI schedule to the category of health facilities that offer the second dose:

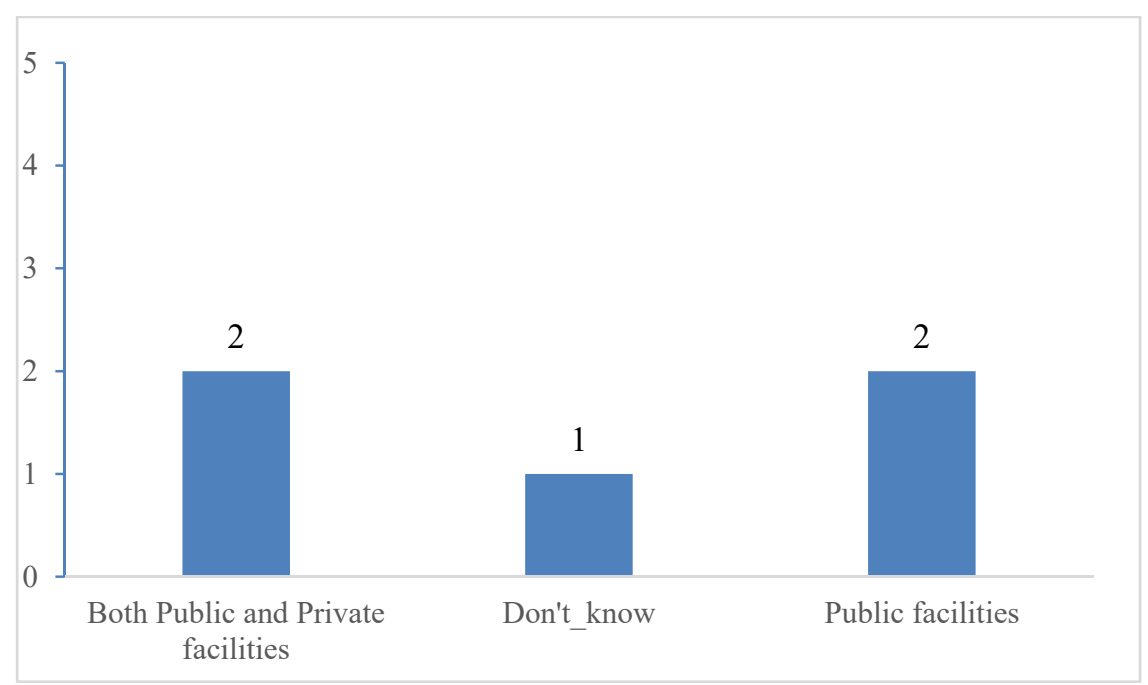

Figure 2. LGAs with facilities that offer a second dose of measles as part of their RI schedule $(n=5)$

All 5 LGAs that reported offering a second dose of measles also reported receiving the vaccines for administering MCV2 from the State Immunization Programme. During Measles campaigns/SIAs, all LGAs reported that data for a second dose of measles is recorded as part of the campaign data. Table 4 shows the factors given by the LGA respondents that affect the quality of measles data. 
Table 4. LGA response to issues associated with reporting quality measles data

\begin{tabular}{lcc}
\hline Issues associated with reporting quality measles data & Frequency & Percent (\%) \\
\hline Lack of commitment from staff & 2 & 18 \\
No response & 8 & 73 \\
Non availability of some register & 1 & 9 \\
Total & $\mathbf{1 1}$ & $\mathbf{1 0 0}$ \\
\hline
\end{tabular}

\subsection{Capacity Development}

All the respondents in the health facilities visited (both public and private) confirmed to have been trained on RI data management. Table 5 below depict the response of the health facilities to when the training was held and the type of trainings that were provided.

Table 5. Response to when the last RI data management training was held for health facility staff and the type of training in Gombe State $(\mathrm{n}=22)$

\begin{tabular}{lcc}
\hline When was the last RI data management training? & Private Facility (3) & Public Facility (19) \\
\hline 1 month ago & $0(0 \%)$ & $4(21.6)$ \\
2 to 3 months ago & $3(100 \%)$ & $10(52.6 \%)$ \\
More than 4 months ago & $0(100 \%)$ & $5(26.3 \%)$ \\
\hline Type of training provided & & \\
Data Analysis \& Use & $0(0 \%)$ & $1(5.3 \%)$ \\
Data Recording & $3(100 \%)$ & $16(84.2 \%)$ \\
Data Reporting & $0(0 \%)$ & $2(10.5 \%)$ \\
\hline
\end{tabular}

All LGAs reported that the health facility staffs have been trained on RI data management. The response to the period of training can be seen below:

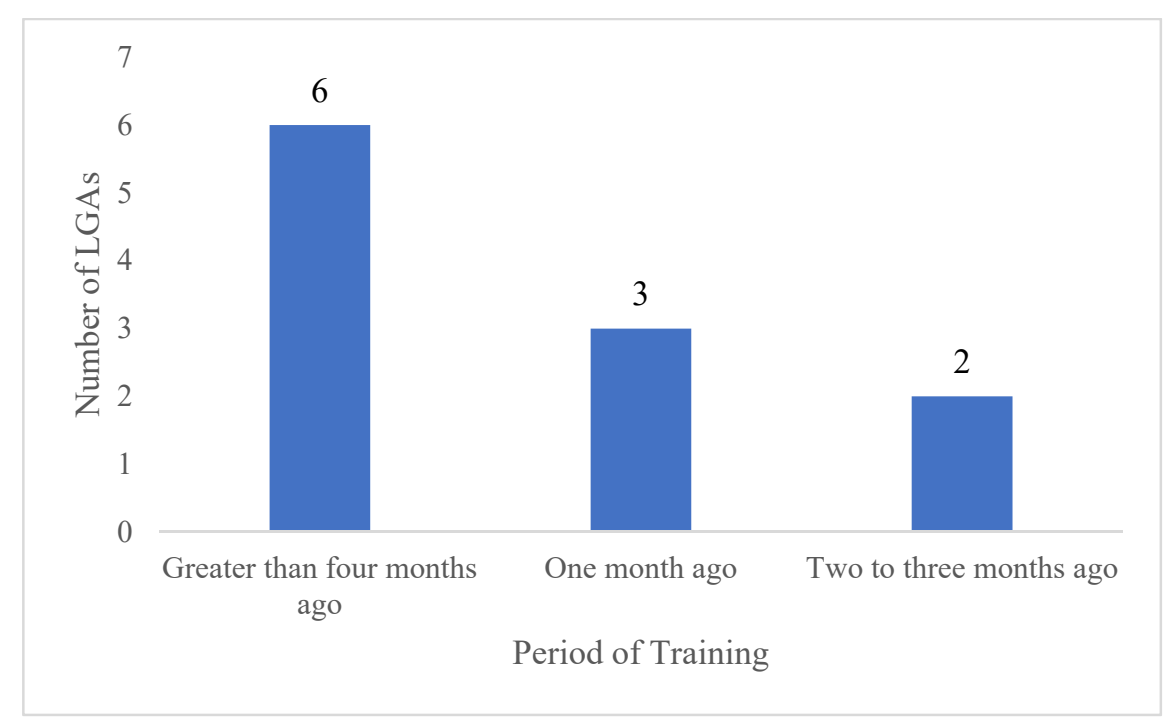

Figure 3. Period of last RI data management training in Gombe State, $2018(\mathrm{n}=11)$

All LGAs reported that the focus of the RI data management training was data recording. Out of the 11 sampled LGAs, 10 (91\%) reported holding LGA data validation meetings. These 10 LGAs also reported that data consistency (between tally sheet, Health facility monthly summary form \& NHMIS monthly summary forms, 
and between vaccines doses opened and administered) is the focus of discussion during the LGA data validation meetings.

\section{Discussion}

From the findings of the study, it was evident that there is still some confusion on whether or not to record children aged 18-23 months who hadn't previously received the first dose of measles under the Measles 2 column of the immunization tally sheets as seen in the results which shows that 2 public health facilities out of the 12 that reported offering a second dose of measles, reported recording under children aged 18-23 months who didn't receive a first dose at age 9 months. These issues can be linked to a poor data use culture and feedback mechanism among health workers. It has been postulated that health workers often do not see the value of the data they collect due to a lack of understanding of understanding of data use, poor feedback mechanism between the health workers and their line managers, among others (Bloland \& MacNeil, 2019). It is expected that the immunization data should be reviewed regularly by the LGA Immunization team to identify issues with the quality of data generated and implement the necessary corrective actions. The issues identified in table 4 by the respondents as the possible reasons for reporting MCV2 can be addressed through the conduct of regular, data quality-focused review meetings at the health facility, LGA and State levels. These meetings should also include data validation sessions where health facility workers are expected to come with the data tools to the LGA secretariat for data validation and verification), however, the sub-optimal quality of immunization data noticed in the LGAs, health facilities and DHIS2 suggest that little or no data validation and review is conducted. These meetings are also supposed to provide a platform for mentoring the health workers on proper documentation practices in the immunization programme, including how to capture children immunized with measles vaccine. The inability of the LGA team to review the DHIS2 data and validate its quality is also associated with capacity gaps noticed during the study. This is also buttressed by the fact that $54.4 \%$ of the LGAs reported that the last training on RI data management received was more than 4 months ago (some going back 2-3 years). Issues of data entry errors and wrong recording/reporting can also be corrected through enhanced supportive supervision techniques that involve on-the-job training of health facility staff, LGA Officers and State level RI Officers. On-the-job training during supportive supervision visits provide the opportunity for the health workers to implement what has been learnt real-time e.g. providing mentoring to health workers during RI sessions. Lessons learnt from previous on-the-job training exercises show that this is a more effective method of capacity building than the usual "classroom" training method. There is also a need to restructure the way data validation meetings are currently being conducted at the LGA level. Even though the LGA team reported that issues of data quality constitute the major talking points during these meetings, the reality is that the time allotted for discussing such issues and other issues of RI data quality aren't sufficient to address them, thus having little or no impact on the quality of data submitted routinely.

During this study, interactions with the key RI stakeholders at the State level showed that there is a disconnect between the State and the LGA level in terms of RI service delivery. State level RI officers need to do more to improve the quality of RI data generated across all levels of RI service delivery in the States. State officers should be assigned to monitor the RI review/data validation meetings at the LGA level. This will go a long way in ensuring that issues of RI data quality are exhaustively discussed, and the meetings are conducted with the highest level of professionalism. DHIS2 RI data review at the State level needs to be strengthened. All key RI stakeholders (including development partners) should be part of the review process to improve coordination and align similar project interests. A deliberate attempt should be made by State immunization teams to provide regular feedback to the lower levels of health service delivery on key performance indicators for immunization, to promote data ownership and use for decision-making.

\subsection{Study Limitations}

Data from past measles campaigns/SIAs in Gombe State could not be obtained to establish the correlation in terms of the timeline between the erroneous MCV2 data entered into the DHIS2 platform and the data generated during the campaigns, for the period under review. It was also difficult to sample an equal number of private and public health facilities because the public health facilities outnumber the private health facilities in the State. Not all facility health workers contacted for the interview consented. Most of the health facilities visited had missing records of the Immunization tally sheets and/or the NHMIS monthly summary forms for some months within the period under review (January 2015 - December 2017), thus making it difficult to pin point the source of MCV2 data attributed to the health facilities on the DHIS2 platform.

\section{Conclusion}

Routine immunization data quality remains a remains focal point of discussion for key health stakeholders and 
policy makers in Nigeria, however, the resources needed for its effective implementation at all administrative levels are limited. A lot of focus has been placed on data reporting, with little or no emphasis on data quality. Health workers need to be equipped with the right knowledge and skills to generate quality data. Resources need to be channeled to capacity development of health workers through regular refresher trainings on RI data management and on-the-job mentoring during supportive supervision visits. At the health facility level, the following activities should be conducted during routine data management activities and data quality supportive supervision visits from the LGA team:

1) Data consistency checks between all RI registers, monthly summary forms and the corresponding data generated from the DHIS2 platform.

2) On the job mentoring on how to correctly record RI data, including measles data. The health facility workers should be sensitized on the antigens given routinely in the routine immunization schedule and the antigens yet to be introduced into the schedule, including MCV2.

Regular RI data review meetings should be conducted at the State and LGA levels to harmonize RI data coming in from different sources and levels. Interventions such as improving access to routine immunization services in rural areas, refurbishing health facilities, providing cold chain equipment, providing vaccines to clients at almost no cost, among others have yielded significant results. Despite the successes recorded by these innovations, the results of the 2016/2017 Multiple Indicator Cluster Survey (MICS)-National Immunization Coverage Survey (NICS) uncovered huge discrepancies between the administrative RI data and what was obtainable in the field. This realization has resulted in the harmonization of efforts by development partners towards strengthening RI data management, including improving data quality. Unfortunately, all data quality-driven interventions are partner-driven, with sub-optimal contribution from the Government of Nigeria towards ownership and sustainability.

The quality of health data has a huge impact on the enactment and implementation of health policies by the Government and key stakeholders, who must rely on data to make key decisions. It is only through strengthening RI data management at the lowest levels that the country can ensure a robust, effective and vibrant routine immunization system.

\section{Acknowledgements}

University of Roehampton, Gombe State Ministry of Health, African Field Epidemiology Network.

\section{Competing Interests Statement}

The authors declare that there are no competing or potential conflicts of interest.

\section{References}

Biellik, R. J., \& Davis, R. (2017). The new World Health Organization recommendation on the 2-dose measles vaccine schedule and the way forward in African region. Pan African Medical Journal, ARTVOL. https://doi.org/10.11604/pamj.supp.2017.27.3.11611

Bloland, P., \& MacNeil, A. (2019). Defining \& assessing the quality, usability, and utilization of immunization data. BMC Public Health, 19(1), 380. https://doi.org/10.1186/s12889-019-6709-1

Dabbagh, A., Laws, R. L., Steulet, C., Dumolard, L., Mulders, M. N., Kretsinger, K., \& Goodson, J. L. (2018). Progress Toward Regional Measles Elimination-Worldwide, 2000-2017. Mmwr Morbidity \& Mortality Weekly Report, 67(47), 7.

DHIS2. (2018). Dashboards app. Retrieved July 16, 2019, from https://dhis2nigeria.org.ng/dhis/dhis-web-dashboard/

Fatiregun, A. (2013). Accuracy and Quality of Routine Immunisation Data Monitoring System in two South-Eastern Districts of Nigeria. The Nigerian Health Journal, 13(2), 7.

Githinji, S., Oyando, R., Malinga, J., Ejersa, W., Soti, D., Rono, J., \& Noor, A. M. (2017). Completeness of malaria indicator data reporting via the District Health Information Software 2 in Kenya, 2011-2015. Malaria Journal, 16(1). https://doi.org/10.1186/s12936-017-1973-y

Ibrahim, B. S., Usman, R., Muhammed, Y., \& Okunromade, O. F. (2017). Burden of Measles in Nigeria: A five-year review of Measles case based Surveillance data from 2012-2016. 18. Retrieved from https://www.researchgate.net/publication/316511631_Burden_of_Measles_in_Nigeria_a_five-year_review_ of_Measles_case_based_Surveillance_data_from_2012-2016 
Kiberu, V. M., Matovu, J. K., Makumbi, F., Kyozira, C., Mukooyo, E., \& Wanyenze, R. K. (2014). Strengthening district-based health reporting through the district health management information software system: The Ugandan experience. BMC Medical Informatics and Decision Making, 14(1). https://doi.org/10.1186/1472-6947-14-40

Measles \& Rubella Initiative. (2017, October 26). Substantial decline in global measles deaths, but disease still kills 90000 per year. Retrieved July 15, 2019, from Measles \& Rubella Initiative website: https://measlesrubellainitiative.org/substantial-decline-global-measles-deaths-disease-still-kills-90-000-peryear/

Nsubuga, F., Luzze, H., Ampeire, I., Kasasa, S., Toliva, O. B., \& Riolexus, A. A. (2018). Factors that affect immunization data quality in Kabarole District, Uganda. PLOS ONE, 13(9), e0203747. https://doi.org/10.1371/journal.pone.0203747

Nzaji, M. K., Lungayo, C. L., Kalala, M. N., Ngoie, C. K. M., Makala, J. P., Mwimba, B. L., ... Mwamba, G. N. (2019). Assessment of The Routine Immunization Data Quality In Tshishimbi Health Zone, Democratic Republic Of Congo. 8(03), 5.

Ohiri, K., Ukoha, N. K., Nwangwu, C. W., Chima, C. C., Ogundeji, Y. K., Rone, A., \& Reich, M. R. (2016). An Assessment of Data Availability, Quality, and Use in Malaria Program Decision Making in Nigeria. Health Systems \& Reform, 2(4), 319-330. https://doi.org/10.1080/23288604.2016.1234864

Omoleke, S. A., \& Tadesse, M. G. (2017). A pilot study of routine immunization data quality in Bunza Local Government area: Causes and possible remedies. Pan African Medical Journal, 27. https://doi.org/10.11604/pamj.2017.27.239.11875

UNICEF. (2018). Nigeria Multiple Indicator Cluster Survey 2016-17: National Survey Finding Report, February 2018. Retrieved from https://www.unicef.org/nigeria/sites/unicef.org.nigeria/files/2018-09/NigeriaMICS-2016-17.pdf

World Health Organization [WHO]. (2009). WHO Policy Recommendation on Routine Measles Second Dose (MCV2): Considerations for Removing the Criterion for Introduction. Retrieved from https://www.who.int/immunization/sage/meetings/2016/october/2_MCV2deliberations_YellowBookFinal.p df?ua $=1 \& u a=1$

\section{Copyrights}

Copyright for this article is retained by the author(s), with first publication rights granted to the journal.

This is an open-access article distributed under the terms and conditions of the Creative Commons Attribution license (http://creativecommons.org/licenses/by/4.0/). 